\title{
Leptomeningeal Enhancement Due to COVID-19 on 3D-FLAIR and T1 Black-Blood MR Imaging Sequences
}

W e read with great interest the literature review by Gulko et $\mathrm{al}^{1}$ that included 22 articles reporting the MR imaging brain findings of 126 patients with coronavirus disease 2019 (COVID 19). We agree with the authors that the most frequent diagnosis on MR imaging is acute and subacute infarct, which was reported in 32 of 126 patients. However, because of the different imaging techniques used in the original articles, the frequency of leptomeningeal involvement may be underestimated.

We observed great differences in the reported frequency of leptomeningeal enhancement in the articles included in this review. Helms et $\mathrm{al}^{2}$ reported enhancement in leptomeningeal spaces in 8 patients (68\%). On the other hand, leptomeningeal enhancement was absent (0\%) in several other studies with similar patient samples. ${ }^{1}$ This discrepancy may be attributed to the different imaging techniques used to evaluate leptomeningeal involvement. The contrast-enhanced 3D-FLAIR sequence was used by Helms et al, ${ }^{2}$ while only conventional T1WI postcontrast sequences were performed in several other studies.

In our practice, 3D-FLAIR sequences or 3D T1 black-blood imaging or both are used to clearly demonstrate leptomeningeal enhancement. Leptomeningeal involvement seems to be a relatively common manifestation of CNS involvement in patients with COVID-19. Herein, we present a 68 -year-old woman with COVID-19 who underwent brain MR imaging 2 weeks after extubation, because of confusion and muscle weakness in her limbs. Turbo spin-echo 3D-T1 black-blood and 3D-FLAIR sequences postcontrast administration revealed leptomeningeal enhancement in the left angular gyrus (Fig 1) and on both sides of the right parieto-occipital sulcus (Fig 2). Enhancement was not prominent on conventional (turbo-field echo) 3D-T1 images.

Leptomeningeal enhancement on FLAIR images is known to occur frequently in MS and non-MS inflammatory neurologic conditions, including encephalitis. In their systematic review Zurawski et $\mathrm{al}^{3}$ suggested that contrast-enhanced FLAIR provided as much as 10 -fold sensitivity compared with conventional $\mathrm{T} 1$ sequences in the detection of low concentration of contrast in the subarachnoid space.

- Indicates open access to non-subscribers at www.ajnr.org http://dx.doi.org/10.3174/ajnr.A7104
The increased sensitivity of T1 black-blood imaging in detecting leptomeningeal enhancement has also been reported. Oh et $\mathrm{al}^{4}$ compared contrast-enhanced gradient recalled-echo, contrastenhanced spin-echo, and black-blood imaging for the detection of leptomeningeal carcinomatosis. According to their results, black-blood imaging had a significantly higher sensitivity than contrast-enhanced gradient recalled-echo and contrast-enhanced spin-echo $(97.43 \%, 64.1 \%$, and $66.67 \%$, respectively) in identifying foci of leptomeningeal enhancement.

The possible underlying mechanism of focal leptomeningeal enhancement may include inflammation-related focal disruption of the blood-meninges barrier and associated fibrosis. Pathologist analysis in patients with MS showed leptomeningeal inflammation, which may lead to the up-regulation of reactive fibroblasts and collagen deposition causing local meningeal fibrosis. ${ }^{3}$ Consequently, both fibrosis and inflammation may result in the trapping of low concentration of gadolinium-based contrast medium within the subarachnoid spaces.

Although contrast-enhanced 3D-FLAIR and T1 black-blood imaging are sensitive in detecting leptomeningeal enhancement, they are not routinely performed in patients with COVID-19 in many institutions. Including one or both sequences in MR imaging protocols for patients with COVID-19 with neurologic symptoms may help to avoid underestimation of leptomeningeal and CNS involvement.

\section{REFERENCES}

1. Gulko E, Oleksk ML, Gomes W, et al. MRI brain findings in $\mathbf{1 2 6}$ patients with COVID-19: initial observations from a descriptive literature review. AJNR Am J Neuroradiol 2020;41:2199-203 CrossRef Medline

2. Helms J, Kremer S, Merdji $\mathrm{H}$, et al. Neurologic features in severe SARS-CoV-2 infection. $N$ Engl J Med 2020;382:2268-70 CrossRef Medline

3. Zurawski J, Lassmann H, Bakshi R. Use of magnetic resonance imaging to visualize leptomeningeal inflammation in patients with multiple sclerosis: a review. JAMA Neurol 2017;74:100-09 CrossRef Medline

4. Oh J, Choi SH, Lee E, et al. Application of 3D fast spin-echo T1 blackblood imaging in the diagnosis and prognostic prediction of patients with leptomeningeal carcinomatosis. AJNR Am J Neuroradiol 2018;39:1453-59 CrossRef Medline 


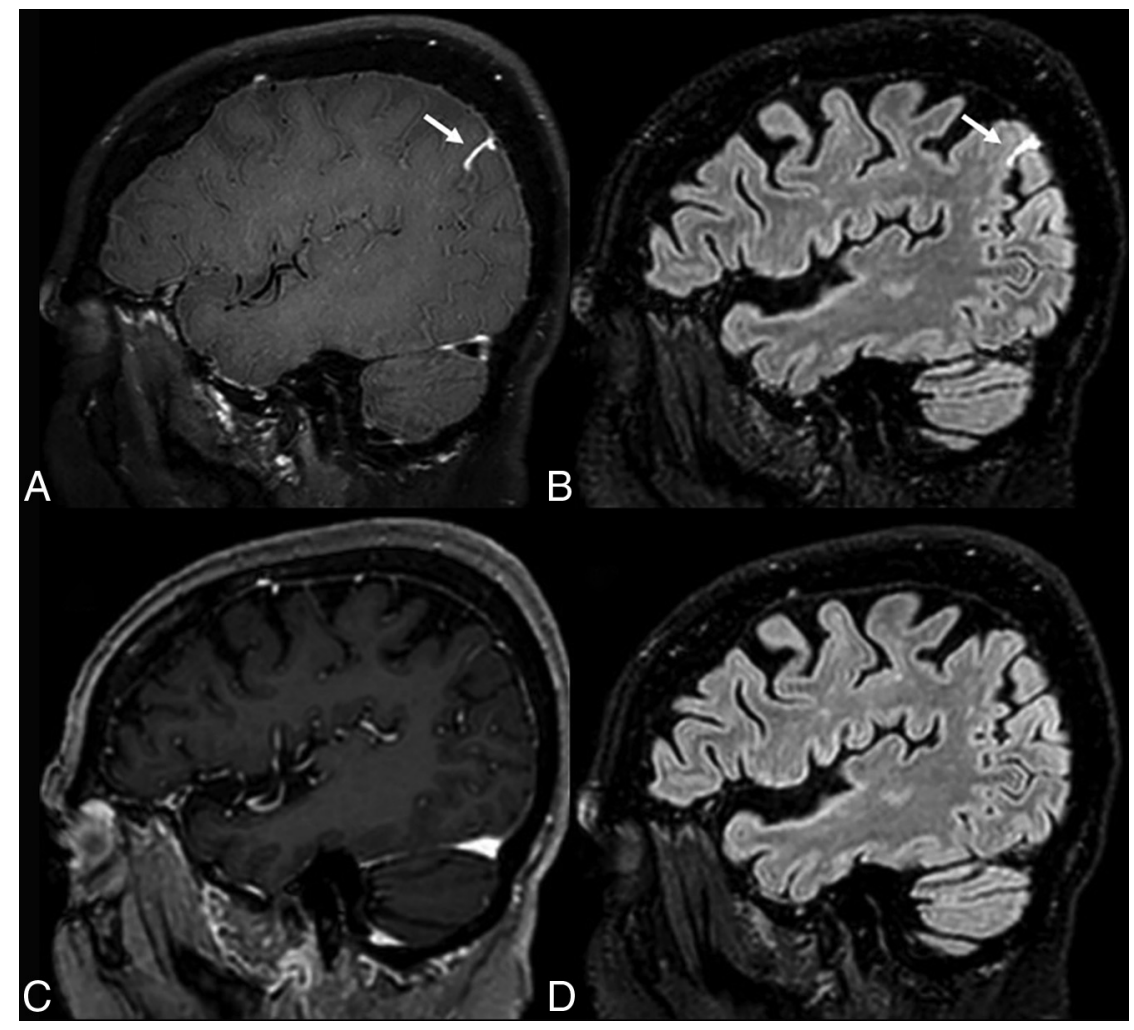

FIG 1. Sagittal 3D turbo spin-echo black-blood T1-weighted $(A)$ and 3D-FLAIR $(B)$ images, both postcontrast administration, reveal leptomeningeal enhancement in the left angular gyrus (arrows, $A$ and $B$ ). Enhancement is not prominent on $T 1$ contrast-enhanced images (C). There are no hyperintensities in this area on the sagittal 3D-FLAIR image without contrast $(D)$.

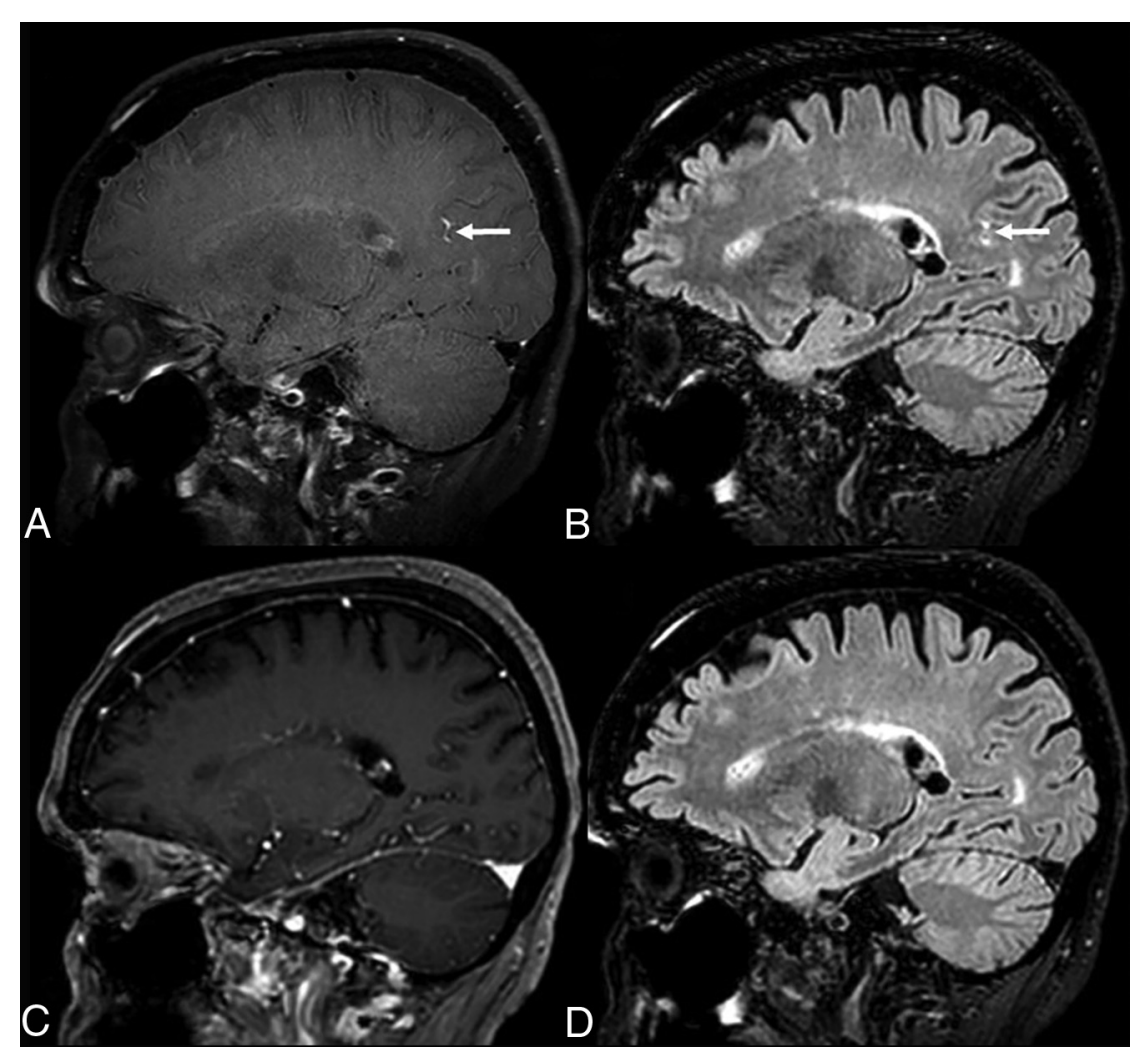

FIG 2. Leptomeningeal enhancement on both sides of the right parieto-occipital sulcus is more evident on sagittal 3D-T7 turbo spin-echo blackblood (A) and 3D-FLAIR (B) images (arrows, $A$ and $B$ ), than on T1 postcontrast (C). No hyperintensities on 3D-FLAIR before contrast administration $(D)$ are noticed in the right parieto-occipital sulcus. 
(1) G. Velonakis

(D). Karavasilis

(D) D.K. Filippiadis

Research Unit of Radiology - 2nd Department of Radiology Medical School, National and Kapodistrian University of Athens

Athens, Greece

(D) M.P, Almyroudi Emergency Department Attikon University Hospital, National and Kapodistrian University of Athens

Athens, Greece

(D). Korompoki Department of Clinical Therapeutics Medical School, National and Kapodistrian University of Athens

Athens, Greece 